\title{
Jugendrecht und Jugenddelinquenz in Japan Japanische Erfahrungen
}

Hiroyuki Kuzuno

Das japanische Jugendrecht erlaubt einen Umgang mit der Jugendkriminalität, über den hier zu Lande niemand mehr (laut) nachzudenken wagt. Die Reaktion auf die Jugendkriminalität ist dort durchweg erzieherisch ausgestaltet. Fast über alle Straftaten entscheidet bis zum 20. Lebensjahr ausschließlich das Familiengericht. Vier Fünftel der Fälle werden eingestellt. Vier Prozent enden mit einer Einweisung in eine Erziehungsanstalt von in aller Regel drei bis zwölf Monaten Aufenthalt. Die deutlich niedrigeren Raten der japanischen Jugendkriminalität sowie die geringen Rückfallquoten werden unter anderem auch auf die informellen und pädagogischen Reaktionen zurückgeführt.

\begin{abstract}
eit einiger Zeit wird in Japan der Ruf nach einem härteren Durchgreifen im Jugendrecht immer lauter, der inzwischen auch die tägliche Praxis erreicht hat. Dieser Wandel ist auf Veränderungen in der japanischen Gesellschaft zurückzuführen. Im Mittelpunkt dieses Artikels steht die Diversion, die in verschiedenen Ländern der Welt im Zusammenhang mit der Reform des jeweiligen Jugend(straf-)rechts thematisiert wird. Die Diversion im japanischen Jugendrecht weist jedoch einige Besonderheiten auf.
\end{abstract}

In Japan war die Diversion nicht immer das vorrangige Ziel. Ein Grund dafür ist die gesetzliche Regelung, wonach Polizei und Staatsanwaltschaft ausnahmslos alle Straftaten Jugendlicher an das Familiengericht abgeben müssen. Es darf also kein Fall außerhalb des familiengerichtlichen Verfahrens eingestellt werden. Jedoch werden nahezu 40\% aller Fälle (ohne Verkehrsdelikte) auf dem Wege des sog. Summary Referral (summarische Abgabe) an das Familiengericht weitergeleitet. Dabei sammelt die Polizei die monatlich anfallenden Bagatellverfahren und übergibt sie en bloc dem Familiengericht, das diese Verfahren in der Regel ohne weitere Untersuchung oder Anhörung einstellt. Die summarische Abgabe wirkt daher de facto wie eine Diversionsmaßnahme.

Der andere Grund ist, dass sich auch die durch Bewährungshelfer geleistete Jugendhilfe im Familiengerichtsverfahren wie eine Diversionsmaßnahme auswirkt. So werden mehr als $80 \%$ der Fälle, die im Wege der regulären Abgabe zum Familiengericht gelangen, auf Grund des Eingreifens der Jugendhilfe vor oder auch nach der gerichtlichen Verhandlung eingestellt. Eine solche Vorgehensweise entspricht dem vom japanischen Jugendgesetz vorgegebenen Ziel, der altersadäquaten Entwicklung der Jugendlichen Rechnung zu tragen.
Nach einem Überblick über das japanische Jugendrecht (1.) wird der gesetzliche Hintergrund und die Praxis der Diversion im Hinblick auf die sozialstaatlich begründete Resozialisierungsfunktion sowie die Funktion der Kriminalitätskontrolle des Jugendrechts untersucht (2.). Sodann wird der aktuelle kriminalpolitische Trend $\mathrm{zu}$ hartem Durchgreifen analysiert (3.).

\section{1. Überblick über das japanische Jugendrecht}

Kurz nach dem zweiten Weltkrieg gingen gravierende gesellschaftliche Umwälzungen mit einer großen Welle der Jugendkriminalität einher. Japan bekam eine neue Verfassung, in der Jugendhilfe und Ausbildung der Kinder sowie die Menschenrechte in den Mittelpunkt gerückt wurden. 1948 wurde ein völlig neues Jugendgesetz erlassen, welches das alte Gesetz von 1922 ersetzte.

\subsection{Zielsetzung und gerichtliche Zuständigkeit}

Dieses neue Jugendgesetz orientierte sich am USamerikanischen Standard Juvenile Court Act von 1943, das auf dem Prinzip des parens patriae beruht. Das Gesetz betonte die Resozialisierungsfunktion des Jugendrechts, während Strafverfahren und strafrechtliche Sanktionen als nicht geeignete Mittel zur Lösung des Problems der Jugendkriminalität angesehen wurden.

Zugleich wurden die Familiengerichte gegründet. Dabei handelt es sich um justizförmige Institutionen, denen ein professioneller Richter vorsitzt. Das Familiengericht ist zuständig in Familiensachen sowie für Straftaten Jugendlicher. Es nimmt daher die zentrale Rolle im japanischen Jugendrecht ein. In jeder von Japans 50 Präfekturen gibt es ein Familiengericht und mehrere Nebenstellen.
In $\S 1$ des Jugendgesetzes wird als dessen Ziel die altersadäquate Entwicklung der Jugendlichen genannt. Das Jugendgesetz regelt die Erziehungsmaßnahmen zur Erreichung dieses Ziels sowie das Verfahren vor dem Familiengericht. Nach $\S 3$ ist das Familiengericht in den folgenden Fällen zuständig: (1) Jugendliche Straftäter, (2) Kinder unter 14 Jahren, die strafrechtliche Normen verletzt haben ${ }^{1}$, (3) Jugendliche unter 20 Jahren, bei denen aufgrund ihrer Persönlichkeit oder sonstigen Umständen eine hinreichende Wahrscheinlichkeit besteht, dass sie eine Straftat begehen werden (potentielle Straftäter).

Nach dem Kinderwohlfahrtsgesetz sind für Fälle im Zusammenhang mit Kindern unter 14 Jahren grundsätzlich die Jugendämter zuständig. Nur wenn diese entscheiden, einen solchen Fall an das Familiengericht abzugeben, damit dieses Erziehungsmaßnahmen anordnen kann, befasst sich das Familiengericht mit Straftaten von Kindern unter 14 Jahren. Im Jahr 2002 betrug die Zahl solcher Fälle 357. Die Zahl der Fälle potentieller jugendlicher Straftäter war im Jahr 2002 mit 1.061 ebenfalls sehr gering. In den allermeisten jugendspezifischen Fällen vor den Familiengerichten geht es um jugendliche Straftäter. Sie stehen im Mittelpunkt der folgenden Ausführungen.

\subsection{Die Entwicklung der Jugendkriminalität in Japan}

Die japanische Jugendkriminalitätsrate ist im Vergleich mit anderen industrialisierten Ländern sehr niedrig und betrug im Jahre 20021.506 Tatverdächtige pro 100.000 Jugendliche ${ }^{2}$. Nach der polizeilichen Kriminalstatistik hat der Anteil der tatverdächtigen Jugendlichen in den letzten Jahren zugenommen. Die Polizei nennt diese Entwicklung »die vierte Welle der Kriminalität« nach dem Zweiten Weltkrieg. 


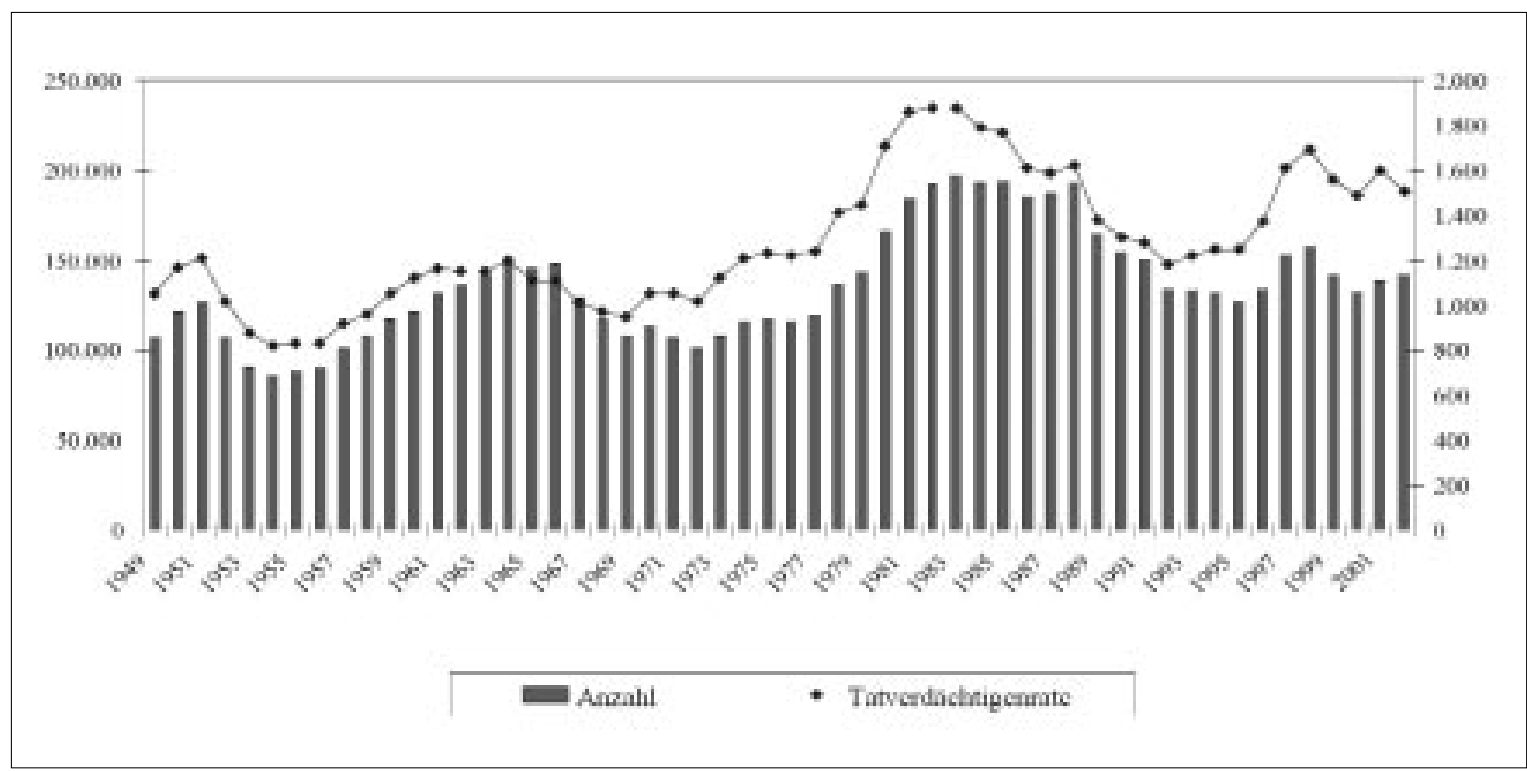

Quelle: White Paper on Police, 2003, p. 200.
In nahezu 87\% der Fälle geht es um Diebstahl oder Unterschlagung (Tab. 1). Dementsprechend hat ein Rückgang oder Anstieg der Zahl der Jufolgt werden, einen großen Einfluss auf die Entwicklung der Zahl der jugendlichen Straftäter insgesamt. Schwankungen der Tatverdächtigenbelastungszahl beruhen weiterhin auf der polizeilichen Verfolgungspraxis gegenüber Jugendlichen, die gerade bei solchen leichteren Delikten sehr unterschiedlich ist.

Anders als bei den Tötungsdelikten ist die Zahl der Jugendlichen, die wegen eines Raubdeliktes verfolgt wurden, seit 1997 angestiegen. Die Massenmedien präsentierten den Anstieg der Raubdelikte als Beleg dafür, dass die Jugendlichen sehr viel gewalttätiger geworden seien, und riefen nach einem härteren Vorgehen gegen jugendlifentliche und politische Debatte über die Jugendrechtsreform. Auch infolgedessen ist das Jugendgesetz im Jahr 2000 neugestaltet worden.

Weiterhin ist beobachtet worden, dass die polizeiliche Gesetzesanwendung gerade im Hinblick auf schwere Fälle von Jugendkriminalität härter geworden ist, und dass der starke Anstieg der Tatverdächtigenbelastungszahl für Raubdelikte nicht nur auf eine tatsächlich höhere Zahl von Tätern, sondern in erster Linie auf eine veränderte Verfolgungspraxis zurückzuführen ist. So konnte zum Beispiel eine Wegnahme, die eine Körperverletzung des Opfers zur Folge hatte, ab 1997 als »Raub mit Körperverletzung « verfolgt werden, und zwar auch bei nur leichten Verletzungen. Zuvor waren solche Fälle als zwei Delikte - Diebstahl und Körperverletzung - behandelt worden ${ }^{3}$. gendlichen, die wegen eines dieser Delikte verche Straftäter. Es entwickelte sich eine starke öf-

\subsection{Das Verfahren vor dem Familiengericht}

Das polizeiliche Ermittlungsverfahren bei Straftaten Jugendlicher folgt den allgemeinen Regeln des Strafprozessrechts. Das Jugendgesetz und die Regelungen zur polizeilichen Ermittlungsarbeit enthalten zwar einige Bestimmungen für eine besondere Behandlung Jugendlicher, etwa spezielle Arrestund Haftbegrenzungen sowie Schutzbestimmungen bei polizeilichen Befragungen. Es bestehen aber große Zweifel, ob diese tatsächlich einen effektiven Schutz der Jugendlichen gewährleisten können.

Die Polizei gibt leichtere Fälle direkt an das Familiengericht $\mathrm{ab}$, während schwerere Fälle an die
Staatsanwaltschaft weitergeleitet werden. Die Staatsanwaltschaft gibt nach Abschluss der Ermittlungen alle Fälle an das Familiengericht ab. Letztlich gelangen so alle Fälle, in denen es um Straftaten Jugendlicher geht, zum Familiengericht (Abb. 2). Im Gegensatz dazu kann die wachsenen das Verfahren ohne Einbeziehung des Strafgerichtes einstellen, auch wenn ein hinreichender Tatverdacht besteht. 2002 betrug der Anteil der durch die Staatsanwaltschaft eingestellten Strafverfahren (ohne Verkehrsdelikte) 36,0 $\%$, während die Verfolgungsrate bei $55,4 \%$ lag. Des Weiteren kann die Polizei bei leichteren Straftaten Erwachsener davon absehen, die Sache überhaupt an die Staatsanwaltschaft weiterzuleiten. 24,3\% der polizeilich verfolgten Straftaten (ohne Verkehrsdelikte) sind im Jahr 2001 auf diese Weise erledigt worden.

Nach der Abgabe an das Familiengericht werden in allen Fällen Vorermittlungen durchgeführt. Haben sich dabei Verdachtsmomente gegen den Jugendlichen ergeben, kann der Familienrichter den familiengerichtlichen Bewährungshelfer anweisen, den Jugendlichen, seine Eltern sowie andere betroffene Personen zu befragen und andere notwendige Ermittlungen vorzunehmen. Diese so genannte Social Investigation (Sozialanamnese) wird in praktisch allen Fällen durchgeführt. Nach $\S 9$ des Jugendgesetzes sollen so Informationen Staatsanwaltschaft bei Er-

\section{Tabelle 1: Jugendliche Tatverdächtige (ohne Verkehrsdelikte), Japan 2002}

\begin{tabular}{|c|c|c|c|c|}
\hline \multicolumn{3}{|l|}{ Gesamt } & 141.775 & $100,0 \%$ \\
\hline & \multicolumn{2}{|l|}{ Eigentumsdelikte } & 123.835 & $87,3 \%$ \\
\hline & & Ladendiebstahl & 40.511 & $(28,6 \%)$ \\
\hline & & Autodiebstahl & 12.650 & $(8,9 \%)$ \\
\hline & & Fahrraddiebstahl & 14.710 & $(10,4 \%)$ \\
\hline & & Unterschlagung & 34.263 & $(24,2 \%)$ \\
\hline & & Andere Delikte & 21.701 & $(15,3 \%)$ \\
\hline & \multicolumn{2}{|l|}{ Gewaltdelikte } & 17.940 & $12,7 \%$ \\
\hline & & Körperverletzung & 9.140 & $(6,4 \%)$ \\
\hline & & Erpressung/Nötigung & 4.616 & $(3,3 \%)$ \\
\hline & & Totschlag & 80 & $(0,06 \%)$ \\
\hline & & Raub & 1.586 & $(1,1 \%)$ \\
\hline & & Andere Delikte & 2.518 & $(1,8 \%)$ \\
\hline
\end{tabular}

Quelle: White Paper on Police, 2003, p. 218 
über das Verhalten, den Werdegang, die Persönlichkeit und die Umgebung des Jugendlichen, seiner Eltern oder anderer betroffener Personen gesammelt werden. Dabei soll nach Möglichkeit auch medizinischer, psychologischer, pädagogischer, soziologischer und anderer Sachverstand herangezogen werden. 2001 betrug die Zahl der familiengerichtlichen Bewährungshelfer 1533, wovon die Hälfte für jugendliche Straftäter verantwortlich ist.

Der Familienrichter kann die Verhandlung vertagen und den Jugendlichen unter vorläufige Bewährung stellen, die vom familiengerichtlichen Bewährungshelfer überwacht wird. Darüber hinaus kann der Familienrichter den Jugendlichen in ein Classification Centre einweisen, wo Untersuchungen zur delinquenten Prädisposition oder zum psychischen Befinden durchgeführt und die Jugendlichen dementsprechend klassifiziert werden können. Derartige Unterbringungsanordnungen wurden 2002 in 9,2\% der Fälle (ohne Verkehrsdelikte) erteilt. ${ }^{4}$ Die Unterbringungsdauer ist grundsätzlich auf 4 Wochen begrenzt. Nach dem revidierten Jugendgesetz von 2000 kann sie in Ausnahmefällen auf bis zu 8 Wochen ausgedehnt werden, wenn dies zur Beurteilung des Jugendlichen notwendig ist.

Die Sozialanamnese hat zwei Funktionen. Zum einen sollen Informationen zum sozialen Hintergrund gesammelt und analysiert werden. Der Familienrichter entscheidet auf Grundlage des Berichts des familiengerichtlichen Bewährungshelfers, welche Erziehungsmaßnahme für den Jugendlichen geeignet ist. Zum anderen soll der Bewährungshelfer aber auch selbst unterstützend und erzieherisch wirken. Während der Untersuchung kann er Anweisungen und Ratschläge geben oder dem Jugendlichen und seinen Eltern Hilfe zur Bewältigung möglicher Probleme anbieten, um Rückfälle des Jugendlichen zu verhindern. Er überwacht die ergriffenen unterstützenden und erzieherischen Maßnahmen und erstellt abschließend einen Bericht. Der Bewährungshelfer erfüllt daher bei Durchführung der Sozialanamnese auch Aufgaben der Jugendhilfe.

Auf Grundlage der Berichte des Bewährungshelfers bzw. des Classification Centre entscheidet der Familienrichter darüber, ob eine Verhandlung stattfinden soll. Gelangt er zu dem Ergebnis, dass es keinen hinreichenden Tatverdacht für eine Straftat gibt oder dass eine Verhandlung für den Jugendlichen ungeeignet wäre, stellt er das Verfahren ohne Verhandlung ein.

Die Verhandlung ist höchst informeller Natur. Nach $\S 20$ Abs. 1 des Jugendgesetzes soll sie in friedvoller und freundlicher Atmosphäre durchgeführt werden. Die Öffentlichkeit, insbesondere die Medien, und sogar die Opfer sind von der Verhandlung ausgeschlossen. Der Jugendliche, seine Eltern und mögliche Begleiter werden zu der Verhandlung vorgeladen, und auch der Bewährungs-
Abbildung 2: Japanisches Jugendrechtverfahren

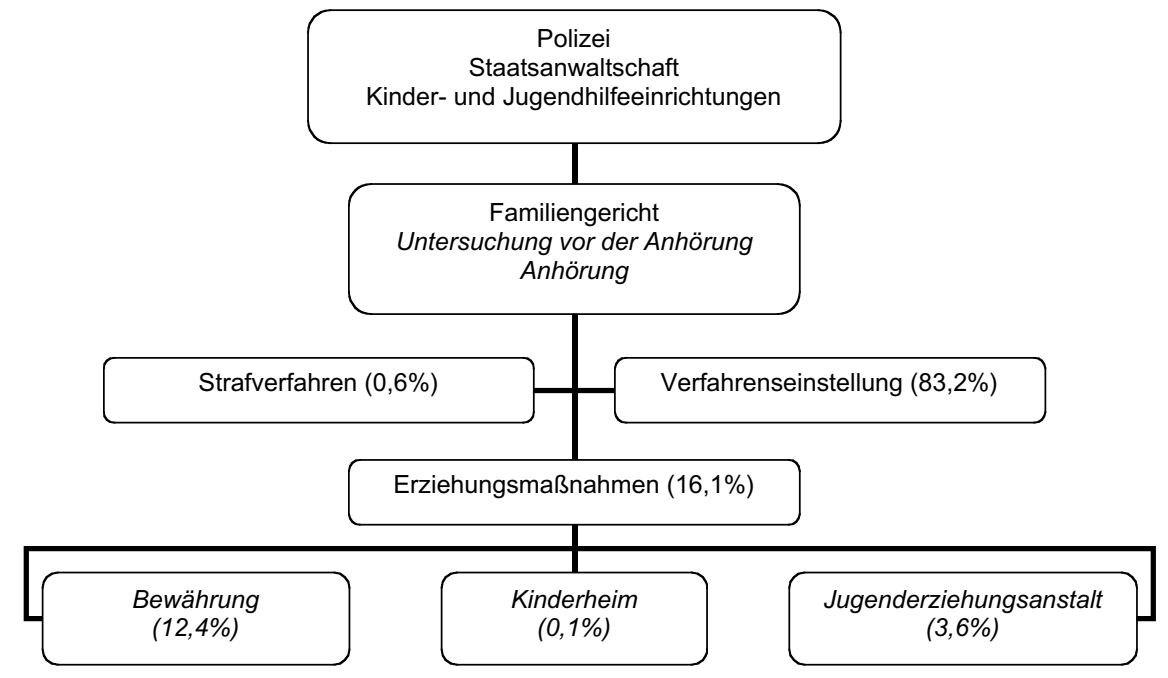

helfer hat anwesend zu sein. Außerdem kann es Lehrern, Arbeitgebern etc. gestattet werden, der Verhandlung beizuwohnen. Die Staatsanwaltschaft darf nur mit besonderer Genehmigung anwesend sein, die vom Familienrichter nur in äußersten Ausnahmefällen erteilt wird. Der Jugendliche oder seine Eltern können einen Begleiter benennen, der üblicherweise Anwalt ist. Im Jahr 2002 ist jedoch nur in 5,7\% der Fälle (ohne Verkehrsdelikte) ein solcher Begleiter benannt worden. Auch wenn dies im Vergleich zu früheren Jahren eine recht große Steigerung bedeutete, ist dies immer noch das schwerwiegendste Problem im Hinblick auf die Rechtsstaatlichkeit des jugendrechtlichen Verfahrens vor dem Familiengericht.

Der Familienrichter stellt das Verfahren auch am Ende der Verhandlung ein, wenn es keine hinreichenden Beweise für eine Straftat gibt oder er zu dem Schluss gelangt, dass Erziehungsmaßnahmen nicht erforderlich sind. Kommt er zu der Auffassung, dass das Verfahren nicht eingestellt werden kann, so kann er Erziehungsmaßnahmen anordnen. Mögliche Maßnahmen sind die Einweisung in eine Jugenderziehungsanstalt, die Einweisung in ein Kinderheim oder die Anordnung der Bewährung.

Schließlich besteht die Möglichkeit, die Sache an die Staatsanwaltschaft weiterzuleiten. Nur in diesem Fall kann ein Jugendlicher vor ein Strafgericht gelangen und als Straftäter verurteilt werden. Allerdings geschieht dies nur in Ausnahmefällen und betraf im Jahr 2002371 Jugendliche (ohne Verkehrsdelikte). Bei Erreichen des 21. Lebensjahres wird die Sache in jedem Fall an die Staatsanwaltschaft weitergeleitet.

\subsection{Die Behandlung der Jugendlichen}

Die Jugenderziehungsanstalten werden vom Justizministerium verwaltet. Zum 1. April 2003 gab es landesweit 53 derartige Anstalten. Die Jugend- lichen werden nach ihrem Alter, der Rückfallwahrscheinlichkeit sowie ihrer physischen und psychischen Verfassung in vier Kategorien eingeteilt. Hinsichtlich der Verweildauer in der Anstalt können drei Konstellationen unterschieden werden. Bei der normalen Kurzzeitbehandlung werden die Jugendlichen über einen Zeitraum von 6 Monaten intensiv betreut. Für Jugendliche mit geringer Delinquenzbelastung gibt es alternativ dazu eine spezielle, 2- bis 3-monatige, Kurzzeitbehandlung in einer offenen Einrichtung. Eine Langzeitbehandlung dauert grundsätzlich ein Jahr. Die Behandlungsdauer kann sich jedoch in Ausnahmefällen auf 4 bis 5 Jahre ausweiten, wenn dies aufgrund besonderer psychischer oder anderer Probleme notwendig ist. Diese spezielle Langzeitbehandlung ist 1998 eingeführt worden, nachdem die Behandlungszeit eines 14-jährigen Mörders in Kobe - sie betrug weniger als 2 Jahre - auf heftige Kritik gestoßen war. Manche der Anstalten sind offene oder halboffene Einrichtungen, wobei auch diese durch strenge Disziplin und intensive Trainingsprogramme gekennzeichnet sind.

In den Kinderheimen wird den Kindern Erziehung, Fürsorge, Bildung und sonstige Unterstützung geboten. Bewährung kann Jugendlichen bis zum Alter von 20 Jahren auferlegt werden. Die Bewährung wird von Bewährungshelfern beaufsichtigt, die den offiziellen Einrichtungen der Bewährungshilfe angehören. Die tagtägliche Betreuung und Unterstützung erfahren die Jugendlichen von ehrenamtlichen Bewährungshelfern.

Jugendliche, die strafgerichtlich verfolgt werden, unterliegen grundsätzlich dem gleichen Verfahren wie Erwachsene. Es gelten jedoch einige Sonderregeln für jugendliche Straftäter. So tritt beispielsweise an die Stelle der Todesstrafe die lebenslange Haft, und an die Stelle der lebenslangen Haftstrafe tritt eine zeitige Haftstrafe von 10 bis 15 Jahren. 


\section{5. Änderungen des Jugendgesetzes}

Das Jugendrecht hat zwei Funktionen: Resozialisierung und Kriminalitätskontrolle. Ziele wie Vergeltung und Abschreckung sind im Jugendrecht an sich unzulässig.

In den letzten 20 Jahren ist der Aspekt der Kriminalitätskontrolle in Theorie und Praxis mehr und mehr in den Mittelpunkt gerückt. Damit verbunden sind Forderungen nach härteren Sanktionen und einer Intensivierung der Ermittlungstätigkeit sowie der Berücksichtigung von Vergeltungs- und Abschreckungsgesichtspunkten bei der Entscheidung jugendrechtlicher Fälle. Als ein Ergebnis dieser Entwicklung kam es im Jahr 2000 zu einer Änderung des Jugendgesetzes.

Diese Gesetzesänderung umfasst drei Teilbereiche. Der wichtigste Teil betrifft die Strafverfolgung Jugendlicher. Vor der Neufassung konnte das Familiengericht nur Fälle Jugendlicher über 16 Jahre an die Staatsanwaltschaft abgeben. Dieses Mindestalter ist auf 14 Jahre gesenkt worden. Darüber hinaus ist das Familiengericht nun grundsätzlich bei Jugendlichen über 16 Jahren, die ein Tötungsdelikt oder eine andere vorsätzliche Straftat mit Todesfolge begangen haben, zur Abgabe verpflichtet. Damit ist die Wahrscheinlichkeit für den Jugendlichen, dass sein Fall vor einem Strafgericht verhandelt wird und zu einer Kriminalstrafe führt, erhöht worden.

Zweitens ist mit dem novellierten Jugendgesetz das Ermittlungsverfahren geändert worden. Die Höchstdauer der Untersuchungshaft ist von vier auf acht Wochen ausgedehnt worden. War die Staatsanwaltschaft vor der Gesetzesänderung von der familiengerichtlichen Verhandlung gänzlich ausgeschlossen, kann das Familiengericht der Staatsanwaltschaft nun gestatten, der Hauptverhandlung beizuwohnen, wenn der Jugendliche die Tat bestreitet oder wenn der Fall sehr kompliziert ist.

Drittens sind verschiedene Bestimmungen im Hinblick auf die Opfer eingefügt worden. Sie können vor dem Familienrichter oder dem Bewährungshelfer eine Aussage machen und so ihre Sicht der Tat und des Täters darstellen. Es ist ihnen erlaubt, die Akten des Falles einzusehen, und sie sind über die abschließende Entscheidung zu informieren. Ähnliche Bestimmungen sind kurz vor der Änderung des Jugendgesetzes auch schon in das allgemeine Strafprozessrecht aufgenommen worden.

Für diese Änderung nennt Yokoyama folgende Gründe: (1) eine Veränderung der öffentlichen Meinung nach dem Kobe-Fall, (2) ein v.a. auf die Ausweitung der sozialen Kontrolle sowie härterer polizeilicher Gesetzesanwendung zurückzuführender Anstieg der registrierten Straftaten, (3) Berichte der Massenmedien, die infolge polizeilicher Pressemitteilungen die Zunahme der Jugenddelinquenz sowohl in quantitativer als auch in qualitativer Hinsicht überbewerteten, (4) die Opferrechtsbewegung, sowie (5) eine politische Bewegung zur kriminalisierung Jugendlicher. ${ }^{5}$

All dies sind sicherlich wichtige Einflussgrößen. Nach dem integrativen Konfliktmodell von McGarrell und Castellano ${ }^{6}$ sind neben diesen konkreten kriminalitäts- und strafrechtsbezogenen Faktoren und dramatischen Einzelfällen aber auch strukturelle und kulturelle Faktoren zu berücksichtigen.

Als strukturelle Faktoren für die Änderung des Jugendgesetzes können etwa die Globalisierung der Wirtschaft, der industrielle Wandel, die Verlagerung der Industrie ins Ausland, eine gravierende Rezession, der neoliberale Abbau des Sozialstaats und der sozialen Sicherheit, wachsende Arbeitsplatzunsicherheit, hohe Arbeitslosigkeit und andere Gründe sozialer Entfremdung unter jungen Leuten, zunehmende Raten von Schulabbrechern und ein Anstieg der Kriminalität genannt werden. Als kulturelle Faktoren können etwa die neoliberale Betonung der »Eigenverantwortung « und »Selbsthilfe «, das Vorherrschen einer »winner-or-loser-Kultur «, eine tiefgreifende Zukunftsangst und eine abnehmende Toleranz in der Bevölkerung, ein weit verbreitetes Gefühl der sozialen Unsicherheit sowie eine wachsende Kriminalitätsfurcht angeführt werden.

Diese strukturellen und kulturellen Faktoren sind einhergegangen mit Faktoren, die sich auf das Straf- und Jugendrecht selbst beziehen, wie einer verstärkten öffentlichen Wahrnehmung der Situation der Opfer, öffentlichem Misstrauen gegenüber dem Jugendrecht und der Forderung nach härterem Durchgreifen bei Jugendkriminalität sowie einer extensiven medialen Berichterstattung. Nach einer Reihe schwerer Straftaten Jugendlicher machten zudem einige Parteien das Jugendrecht bei den Wahlen im Jahr $2000 \mathrm{zu}$ einem zentralen Wahlkampfthema, und es kam zu einer außergewöhnlichen Beschleunigung des politischen Prozesses, die noch im selben Jahr in der Gesetzesänderung mündete. Indem es die strafrechtliche Verantwortlichkeit der Jugendlichen und die Bestrafungsmöglichkeiten ausweitet, spiegelt das geänderte Jugendgesetz die vorherrschende »winner-or-loser-Kultur « und andere kulturelle Faktoren wieder.

\section{Diversion und Jugendhilfe im Familien- gerichtsverfahren}

\subsection{Summarische Abgaben durch die Polizei als versteckte Diversion}

Vor Einführung der Familiengerichte hatte die Staatsanwaltschaft nach Überprüfung der Fälle zu entscheiden, welche davon strafgerichtlich verfolgt werden sollten und welche an das Juvenile Hearing Centre abzugeben waren. An diese Einrichtung gelangten die Fälle, in denen die Staatsanwaltschaft eine strafrechtliche Verfolgung für unnötig erachtete.
Heute geht man davon aus, dass auch bei Jugendlichen, die nur leichtere Straftaten begangen haben, Unterstützungsbedarf bestehen kann. Um dies in jedem Einzelfall klären zu können, verlangt das aktuelle Gesetz, dass alle, d.h. auch die leichteren Fälle an das Familiengericht abgegeben werden. Dieses verfügt mit der Bewährungshilfe über die nötigen Ressourcen, um den sozialen Hintergrund der Straftat zu beleuchten und zu entscheiden, welche altersadäquaten Maßnahmen geeignet sind. Polizei und Staatsanwaltschaft sollen keine abschließenden Entscheidungen treffen.

Die Umstände eines jeden Falles derart eingehend zu beleuchten, würde jedoch schnell zu einer Überlastung des Familiengerichts führen. Vor diesem Hintergrund ist im Jahre 1950 durch ein Übereinkommen zwischen dem Obersten Gericht, der Obersten Staatsanwaltschaft und der nationalen Polizeibehörde das bereits skizzierte Verfahren der summarische Abgabe eingeführt worden. Gemäß der Neufassung des Abkommens aus dem Jahr 1969 kann die summarische Abgabe eingesetzt werden, (1) wenn es um Diebstahl, Körperverletzung sowie andere Straftaten geht, die nach dem Erwachsenenstrafrecht mit höchstens drei Jahren Haftstrafe belegt werden könnten, (2) wenn der Vermögensschaden weniger als 5000 Yen beträgt (weniger als 1000 Yen bei Erpressung und Nötigung) oder der Schaden innerhalb von 10 Tagen wiedergutgemacht worden ist, (3) keine Waffe benutzt worden ist und (4) wenn der Jugendliche innerhalb der vergangenen zwei Jahre keinen Eintrag beim Familiengericht hatte, er die Tat nicht abstreitet und nicht in Haft genommen worden ist. Handelt es sich um ein leichteres Vergehen und erachtet die Polizei Erziehungsmaßnahmen für nicht erforderlich, kann sie einen solchen Fall zusammen mit anderen Bagatellverfahren an das Familiengericht abgeben, nachdem sie eine Verwarnung gegenüber dem Jugendlichen oder anderen betroffenen Personen ausgesprochen hat. Nach Eingang beim Familiengericht führt der familiengerichtliche Bewährungshelfer in solchen Fällen keine Sozialanamnese mehr durch. Vielmehr stellt der Familienrichter den Fall nach Durchsicht der polizeilichen Unterlagen ohne Verhandlung ein. Die Zahl der Fälle (ohne Verkehrsdelikte), die im summarischen Verfahren zum Familiengericht gelangten, betrug im Jahr 2002 36,8\%.

Im Zuge einer Untersuchung der Praxis der summarischen Abgaben kam Hattori zu dem Ergebnis, dass sie sich letztlich genauso auswirken, als wenn die entsprechenden Fälle überhaupt nicht an das Familiengericht abgegeben worden seien. ${ }^{7}$ Praktisch alle Fälle, die auf dem Wege der summarischen Abgabe zum Familiengericht gelangen, werden nach kurzer Durchsicht der Akten eingestellt. Zu weitergehenden Untersuchungen oder einer Verhandlung kommt es grundsätzlich nicht. Die summarische Abgabe stellt daher de facto eine Diversion auf polizeilicher Ebene dar. 


\subsection{Diversion und Jugendhilfe im Familienge- richtsverfahren}

Auch ohne die summarische Abgabe werden mehr als 80\% aller Fälle (ohne Verkehrsdelikte) ohne oder nach der familiengerichtlichen Verhandlung auf Grundlage der Sozialanamese des Bewährungshelfers eingestellt. Im Jahr 2002 lagen mehr als $80 \%$ der Verfahrenseinstellungen mit oder ohne Verhandlung erzieherische Begleitmaßnahmen des Bewährungshelfers zugrunde. Der hohe Anteil an Einstellungen ist somit auf die Jugendhilfearbeit während des Familiengerichtsverfahrens zurückzuführen.

Wie dargelegt, kann der Jugendliche auch unter vorläufige Bewährung gestellt werden, die der Bewährungshelfer begleitet und überwacht. In dieser Zeit werden verschiedene erzieherische Maßnahmen ergriffen. In einigen Fällen wird einem Ehrenamtlichen aus der Gemeinde die Bewährungsaufsicht übertragen. Zusätzlich kann der Jugendliche auch in einem Privathaushalt oder einer privaten Einrichtung untergebracht werden. Üblicherweise wird die vorläufige Bewährung für jene Jugendliche angeordnet, die wegen ernsthafter persönlicher Probleme oder Schwierigkeiten in ihrem Umfeld sehr wahrscheinlich in eine Jugenderziehungsanstalt $\mathrm{zu}$ überweisen wären. Die vorläufige Bewährung wird daher auch als »letzte Chance« eingestuft. Zum Abschluss der vorläufigen Bewährung führt der Familienrichter eine Verhandlung durch, und in der Regel stellt er das Verfahren ein oder ordnet eine Bewährungsstrafe an. Die Einweisung in eine Jugenderziehungsanstalt wird normalerweise vermieden. Auch hierin zeigt sich die jugendhilfeorientierte Ausrichtung der Arbeit des Bewährungshelfers.

Ursprünglich ist das Jugendrecht geschaffen worden, um die Jugendlichen vor dem Strafverfahren und vor einer strafrechtlichen Verurteilung zu bewahren, und es funktioniert bis heute so. Das Verfahren stimmt mit dem Gedanken überein, dass Zwangsmaßnahmen und Freiheitsbeschränkungen soweit wie möglich vermieden werden sollten, um eine altersadäquate Entwicklung der Jugendlichen zu gewährleisten.

\section{Härteres Durchgreifen und Wandel der Diversionspraxis}

\subsection{Bedeutungsverlust der familiengerichtli- chen Bewährungshilfe}

Von Anfang an waren die Bewährungshelfer überlastet, da adäquate Jugendhilfe zeit- und arbeitsintensiv ist. Diese zunehmende Betonung der Kriminalitätskontrolle sowie Forderungen nach Vergeltung und Abschreckung haben zu einem Bedeutungsverlust der Resozialisierungsfunktion des Jugendrechts geführt. Dies hat die Diversionspraxis negativ beeinflusst.
1979 ist erstmals offiziell erklärt worden, dass die jugendhilfeorientierte Arbeit des Bewährungshelfers der gerichtlichen Arbeit des Familienrichters unterzuordnen sei, womit die Kriminalitätskontrollfunktion in den Vordergrund gerückt wurde. Das japanische Gerichtswesen ist sehr professionell, zentralisiert, hierarchisch und mit klassischen bürokratischen Strukturen organisiert ${ }^{8}$. Mit der politischen Vorgabe, die Verfahren effizient zu bearbeiten, ist Ende der 70er Jahre großer Druck auf die familiengerichtlichen Bewährungshelfer erzeugt worden, ihre Arbeitsweise zu vereinfachen.

Dieser Druck sowie der Bedeutungsverlust der Resozialisierungsfunktion führten zu verschiedenen Veränderungen ${ }^{9}$. War der Bewährungshelfer ursprünglich als unabhängiger Experte zur Durchführung der Jugendhilfe und zur Berichterstattung an das Familiengericht gedacht, ist er jetzt vollständig in die bürokratische Hierarchie des Landes integriert worden. Im Jahre 1984 wurde ein strenges Aufsichtssystem für die Arbeit der Bewährungshelfer eingeführt. Seit einer Anweisung des Obersten Gerichts aus demselben Jahr können auch solche leichteren Fälle, die im Wege einer normalen Abgabe und nicht durch summarische Abgabe zum Familiengericht gelangen, ohne ausführliche Sozialanamnese eingestellt werden. Schätzungen ergaben, dass heute die Hälfte aller an das Familiengericht abgegebenen Fälle, ohne Sozialanamnese und damit einhergehende erzieherische Arbeit des Bewährungshelfers eingestellt werden.

Der Bedeutungsverlust der Arbeit des Bewährungshelfers spiegelt sich in einem dramatischen Rückgang der Fälle vorläufiger Bewährung wider $^{10}$. Familiengerichtliche Anordnungen von Er-

\section{Abbidlung 3: Neueinweisungen in Jugenderziehungsanstalten und in Classification Centres,} 1952-2002

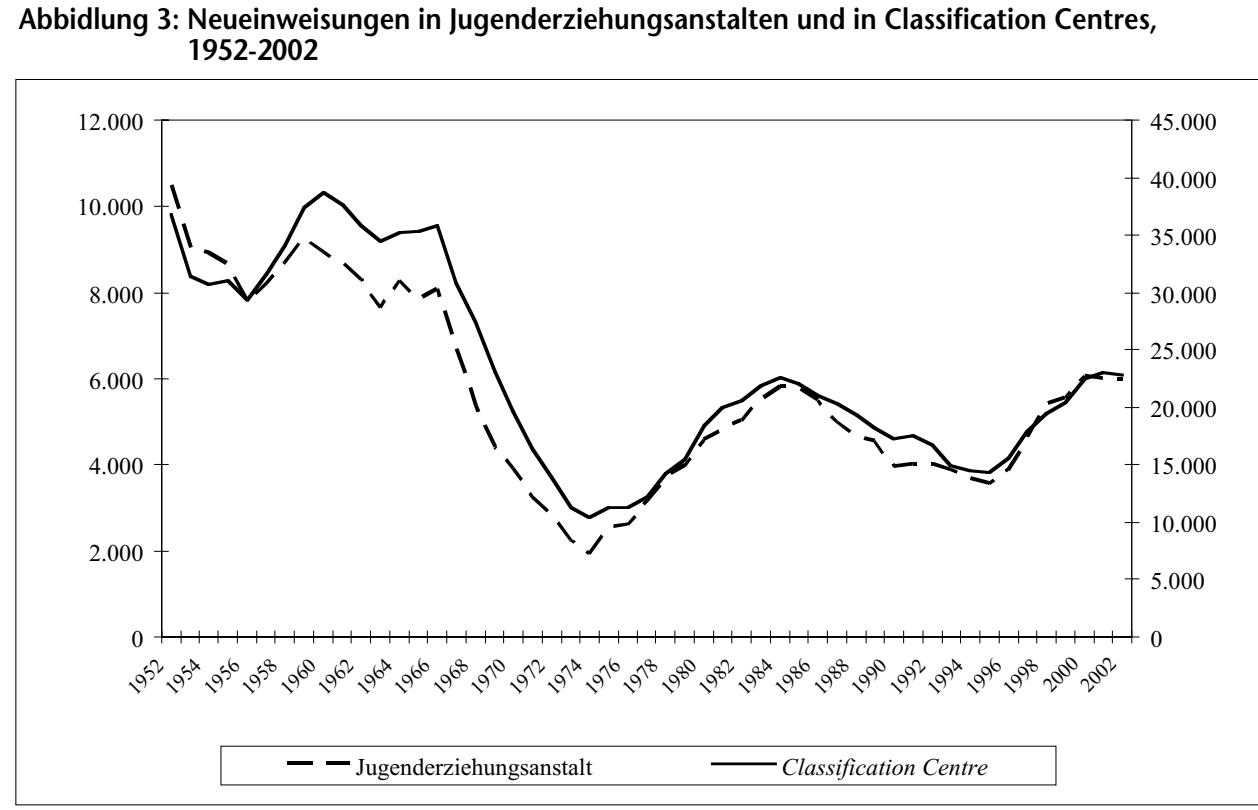

Quelle: White Paper on Police, 2003, p. 230

ziehungsmaßnahmen haben schrittweise zugenommen, während die Zahl der Einstellungen zurückgegangen ist. Die Zahl der Neuzugänge in den Jugenderziehungsanstalten sowie in den Classification Centres ist seit 1996 stark angestiegen (Abb. 3). Zwischen 1995 und 2002 gab es einen 60-prozentigen Anstieg in den Jugenderziehungsanstalten und eine Zunahme um $68 \%$ in den Klassifikationsanstalten, was zu großen Problemen wegen Überbelegung geführt hat.

\subsection{Die praktischen Folgen des geänderten Ju- gendgesetzes und ihr Einfluss auf die Be- währungshilfe}

Vor der Gesetzesänderung im Jahr 2000 ist der Anteil der Fälle, die an die Staatsanwaltschaft abgegeben worden sind, selbst bei schweren Fällen sehr gering gewesen. Da jetzt nach dem neuen $\S 20$ Abs. 2 Jugendgesetz alle Verfahren gegen Jugendliche, denen ein Tötungsdelikt oder eine vorsätzliche Straftat mit Todesfolge zur Last gelegt wird, an die Staatsanwaltschaft abgegeben werden müssen, ist der Anteil der strafrechtlich verfolgten Jugendlichen stark angestiegen. Die Entscheidung solcher Fälle richtet sich de facto primär nach der Schwere der Straftat. Auch hier wird das hohe Gewicht des Gedankens der Kriminalitätskontrolle deutlich.

Das Familiengericht lässt nun auch Aspekte der Vergeltung und Abschreckung bei der Entscheidung der Fälle mit einfließen, was härtere Sanktionen und eine höhere Zahl an Strafverfahren gegen Jugendliche zur Folge hat. Unmittelbar nach der Gesetzesänderung hat das Oberste Gericht entschieden, dass der familiengerichtliche Bewährungshelfer bei der Erstellung seines Berichts nun auch die Auswirkungen der Straftat auf 
Abbidlung 4: Alle an das Familiengericht abgegebenen Jugendkriminalfälle und Summary Referral (summarische Abgabe an das Familiengericht) ohne Verkehrsdelikte, 1970-2002

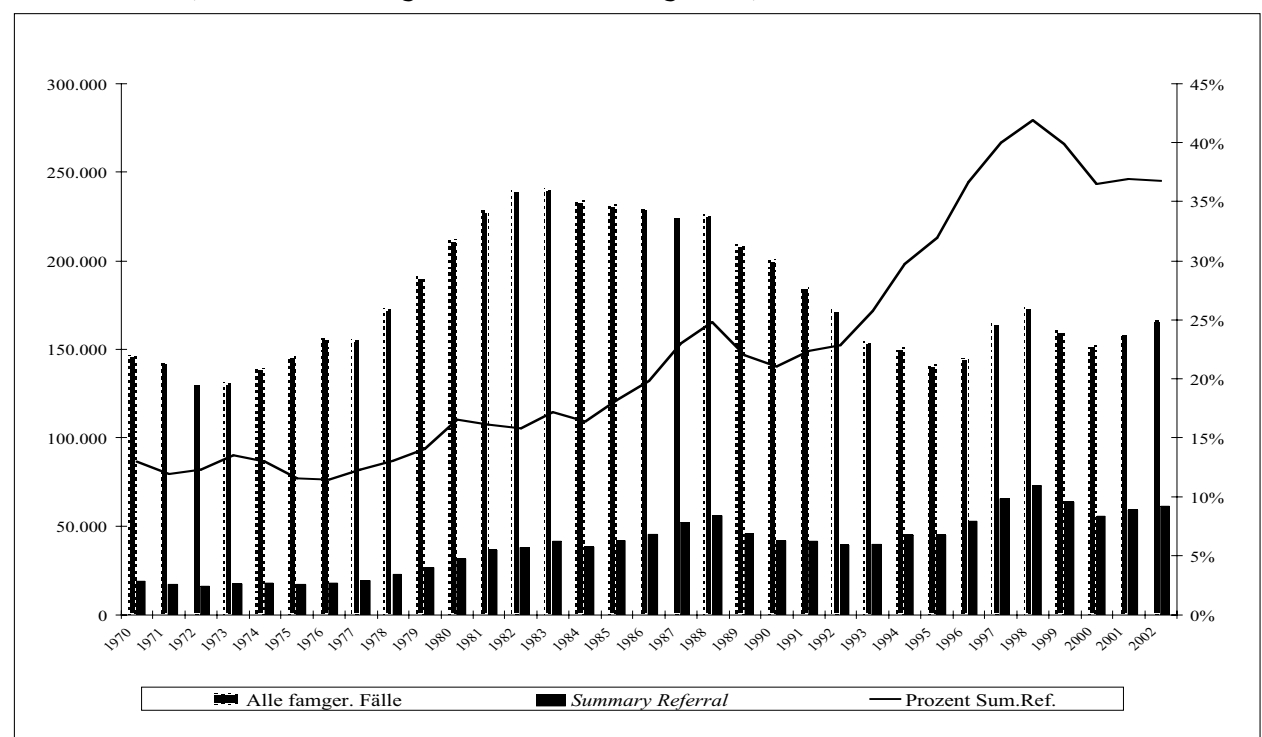

Quelle: Annual Report on Judicial Statistics, 1970-2002

die jeweilige Gemeinde und die Opfer sowie das Vergeltungsbedürfnis von Öffentlichkeit und Opfer sowie den Abschreckungsbedarf untersuchen soll. Dies alles soll sicherstellen, dass ein Großteil der Fälle in einem Strafverfahren mündet. Stellungnahmen $\mathrm{zu}$ Vergeltungs- und Abschreckungsaspekten stehen jedoch in deutlichem Widerspruch zur Jugendhilfeaufgabe des Bewährungshelfers. Nach Angaben von Bewährungshelfern hat dieser Widerspruch für allgemeine Verwirrung über die eigene Rolle gesorgt und die Arbeitsmoral geschwächt.

\subsection{Zunahme der summarischen Abgaben und Bedeutungsverlust der Resozialisie- rungsfunktion}

Während die Zahl der Einstellungen durch das Familiengericht zurückgegangen ist, hat sich die Zahl der summarischen Abgaben der Polizei an das Familiengericht drastisch erhöht (Abb. 4). Da summarische Abgaben de facto wie Diversionsmaßnahmen wirken, scheint diese Ausweitung auf den ersten Blick den Diversionsgedanken zu stärken.

Eine frühzeitige Einstellung des Verfahrens schon auf polizeilicher Ebene kommt denjenigen Jugendlichen zugute, die keine ernsthaften Probleme haben und daher keiner Intervention bedürfen. Sie stimmt also mit dem Ziel altersadäquater Entwicklung überein, da nicht notwendige Interventionen solche Jugendlichen nur unnötigerweise stigmatisieren würden. Dies war neben dem Effekt, dass so eine große Zahl von Verfahren wegen leichterer Straftaten effizient erledigt werden können, der Hauptgrund für die Schaffung des Verfahrens der summarischen Abgabe.

Andererseits gibt es auch Jugendliche, die zwar nur eine leichtere Straftat begangen haben, bei denen aber dennoch ernsthafte Probleme in Bezug auf ihre Persönlichkeit oder ihr soziales Umfeld bestehen und die dementsprechend adäquater Fürsorge und Unterstützung bedürfen. Befasst man sich nur mit der Straftat selbst und ihrer Schwere, so können solche Probleme und Bedürfnisse nicht erkannt werden. Im Hinblick auf die summarischen Abgaben ist fraglich, ob die Polizei derartige Probleme effektiv erkennen kann.

Hatari hat darauf hingewiesen, dass die Entscheidung für oder gegen eine summarische $\mathrm{Ab}$ gabe auf externen Faktoren der Straftat und hierbei in erster Linie auf deren Schwere beruht, und dass die Entscheidung für eine summarische Abgabe dazu führt, dass die Polizei weniger Zeit für die Bearbeitung eines Vorgangs aufwenden muss, etwa für die Anfertigung der Akten. ${ }^{11}$ Dementsprechend werden summarische Abgaben durchgeführt, ohne die Probleme und Bedürfnisse der Jugendlichen sorgsam zu berücksichtigen. Die Fälle, die durch summarische Abgabe zum Familiengericht gelangen, werden praktisch ohne jegliche Hilfe und Unterstützung des Bewährungshelfers für den Jugendlichen eingestellt. Damit beschleunigt die Praxis der summarischen Abgabe, die der Polizei die Hauptentscheidung über den Fortgang des Verfahrens überträgt, den Bedeutungsverlust der Resozialisierungsfunktion des Jugendrechts.

\section{Zusammenfassung}

Eines der Hauptmerkmale des japanischen Jugendrechts ist es, dass alle Fälle zum Familiengericht gelangen und dass ein großer Teil davon infolge der Arbeit des Bewährungshelfers ohne gerichtlich angeordnete Erziehungsmaßnahmen eingestellt wird. Es gibt jedoch eine Tendenz, die
Kriminalitätskontrolle in den Mittelpunkt des Jugendrechts zu rücken, während die Resozialisierungsfunktion ins Abseits gerät. Diese Entwicklung hat die Jugendhilfe durch die familiengerichtlichen Bewährungshelfer geschwächt. Der jüngste Trend zum harten Durchgreifen sowie die Änderung des Jugendgesetzes im Jahr 2000 haben diese Entwicklung weiter beschleunigt und beeinflussen die Diversionspraxis negativ.

Die Ausweitung der Bestrafung Jugendlicher wird nicht zu einem Rückgang schwerer Straftaten führen, da diese in den seltensten Fällen auf einer rationalen Entscheidung beruhen. Die Bestrafung, insbesondere die Inhaftierung in Anstalten des Erwachsenenstrafvollzuges, verhindert die Reintegration straffälliger Jugendlicher und erhöht die Wahrscheinlichkeit eines Rückfalls. Dementsprechend steigt dadurch die Zahl der Verbrechensopfer eher noch an. Jugendliche haben in vielen Fällen aufgrund persönlicher Probleme und Schwierigkeiten in ihrem Umfeld einen großen Bedarf an Erziehung und Fürsorge. Daher rechtfertigen schwere Straftaten Jugendlicher nicht ohne weiteres harte Strafen. Die Intensivierung der Strafverfolgung hat kaum mehr als eine symbolische Wirkung, indem der Öffentlichkeit demonstriert wird, dass etwas gegen das schwerwiegende Problem der Jugendkriminalität getan wird. Dies waren im Übrigen die Hauptgründe dafür, dass ich mich im Jahr 2000 gegen die Änderung des Jugendgesetzes aussprach. ${ }^{12}$

Japan ist bekannt für seine niedrige Kriminalitätsrate. Harada hat aufgezeigt, dass die Kriminalitätsrate Erwachsener im Vergleich zur Kriminalitätsrate Jugendlicher erheblich niedriger ist. Er führt dies vor allem auf eine relativ niedrige Rückfallquote derjenigen Jugendlichen zurück, die in späten Jugendjahren das Jugendrechtsverfahren durchlaufen haben. Nach seiner Auffassung hat das japanische Jugendrecht, begünstigt durch eine positive Entwicklung der wirtschaftlichen Situation und des Arbeitsmarktes, die Resozialisierung straffälliger Jugendlicher gefördert und dazu beigetragen, Rückfälle zu verhindern. ${ }^{13}$ Die Jugendhilfe durch die Bewährungshelfer hat daran einen entscheidenden Anteil, indem sie viele Jugendliche vor formellen Erziehungsmaßnahmen bewahrt und ihren Weg zurück in die Gesellschaft geebnet hat.

Die wichtigste Aufgabe der Jugendrechtsreform ist es daher, die Jugendhilfe wieder zu stärken. Darüber hinaus sollte die erzieherische Verantwortung des sozialen Nahbereiches gefördert werden, um eine bessere Resozialisierung der Jugendlichen, die vor einem formellen Familiengerichtsverfahren bewahrt wurden, $\mathrm{zu}$ erreichen. Auf diesem Wege kann den Jugendlichen eine altersadäquate Entwicklung ermöglicht werden.

Aus dem Englischen übersetzt von Nicole Hilfert und Christian Walburg, Institut für Kriminalwissenschaften, Universität Münster. 
Hiroyuki Kuzuno, Ph. D., ist Professor für Jugendrecht an der Juristischen Fakultät der Ritsumeikan Universität in Kyoto, z. Zt. Visiting Fellow an der London School of Economics und Research Associate am International Centre for Prison Studies, King's College, University of London.

\section{Fußnoten:}

1 Kinder unter 14 Jahren sind nach japanischem Recht strafrechtlich nicht verantwortlich.

2 White Paper on Police 2003, p. 200; in Deutschland 2002: 7.332, Bundeskriminalamt 2003: Polizeiliche Kriminalstatistik 2002, Wiesbaden, S. 97.

3 Hiroyuki Kuzuno: »The Reconstruction of Juvenile Justice«, S. 478-482 (Nihon-Hyoron Sya 2003).

4 Statistiken zum Familiengerichtsverfahren nach 1999 wurden auf der Basis von Fällen ohne summarische Abgabe erstellt. Daher ist es nicht möglich, Zahlen von vor 1999 mit denen danach zu vergleichen.
5 Yokoyama (2003): Revision of the Juvenile Law Toward Partial Criminaliziation in Japan, S. 1547-1551; in: A Manganas (Ed.): Essays in Honour of Alice Yotopoulos-Marangopoulos: Human Rights, Crime, Criminal Policy: Volume B. Athen: Bruylant. Siehe auch Schwarzenegger (2003): The Debate about the reform of the Juvenile Law in Japan, S. 181-191; in: Gesine Foljanty-Jost (Hrsg.): Juvenile Delinquency in Japan. Brill; ders. (2002): Jugendkriminalität und Jugendkriminalpolitik in Japan, in: Reinhard Moss (Hrsg.), Festschrift für U. Jesionek zum 65. Geburtstag, Wien: nw-Verlag, 221-241.

6 McGarrell und Castellano (1993): Social Structure, Crime and Politics: A Conflict Model of the Criminal Law Formation Process; in: William J. Chambliss, Marjorie S. Zatz (Ed.): Making Law 347. Indiana University Press.

7 Hattori (1991): Minor Delinquency, 63 (12) Ho-ritsu Jiho 60-64.

8 Feeley (2002): The Bench, The Bar and the State: Judicial Independence in Japan and the United States, S. 80-81; in: Malcolm M. Feeley und Setsuo Miyazawa (Hrsg.): The Japanese Adversary System in Context. Palgrave.
9 Fukuda (1990): A Critical Analysis of the Juvenile Justice System in Japan, S. 18; in: Hitotsubashi Journal of Law and Politics 1, 7.

10 Yokoyama hat darauf hingewiesen, dass es aufgrund ökonomischer, sozialer und kultureller Veränderungen schwieriger geworden ist Ehrenamtliche zu rekrutieren. Seiner Ansicht nach hat der Rückgang der Fälle vorläufiger Bewährung mit diesen Veränderungen in der japanischen Gesellschaft $\mathrm{zu}$ tun (Yokoyama (2002): Juvenile Justice and Juvenile Crime: An Overview of Japan; in: John Winterdy (Hrsg.): Juvenile Justice Systems: International Perspectives 2. Auflage, Canadian Schloars' Press.

11 Hattori, a.a.O., S. 62.

12 Eine Erklärung gegenüber dem Rechtsausschuss des Parlaments vom 26. Oktober 2000.

13 Harada (2001): Juvenile Delinquency and Adult Crime in Japan, S. 20-33; in: Masami Yajima (Hrsg.): Sociology and Daily Life 2. Ausgabe, Gakubun Sha

\section{Früh übt sich:}

\section{Der Konsum psychoaktiver Substanzen bei Jugendlichen im europäischen Vergleich}

Matthias Richter

Im Sommer diesen Jahres haben Forschungsteams aus fast allen europäischen Ländern bereits zum sechsten Mal die Ergebnisse der Studie »Health Behavior in School-aged Children (HBSC)《 vorgelegt.1 Die HBSC-Studie ist ein internationales kooperatives Forschungsvorhaben, das von der Weltgesundheitsorganisation (Regionalbüro für Europa) unterstützt wird. Ziel der alle vier Jahre durchgeführten Untersuchung ist es, zu einem erweiterten Verständnis gesundheitsbezogener Einstellungen und Verhaltensweisen junger Menschen beizutragen und die Bedingungen ihrer Entwicklung zu untersuchen. In der aktuellen Studie (2001/02) wurden insgesamt über 160.000 Kinder und Jugendliche im Alter von 11,13 und 15 Jahren zu unterschiedlichsten Aspekten ihrer Gesundheit und ihres Gesundheitsverhaltens befragt. Deutschland ist - vertreten durch das Land Nordrhein-Westfalen - seit 1994 an der Studie beteiligt. Die aktuelle Studie fand erstmals nicht nur in Nordrhein-Westfalen, sondern auch in den Bundesländern Berlin, Hessen und Sachsen statt.

$\mathrm{D}$ as gesundheitsrelevante Verhalten und hier insbesondere der Substanzkonsum bilden einen wichtigen Schwerpunkt der Studie. Der Substanzkonsum im Jugendalter verdient besondere Aufmerksamkeit, da Jugendliche in dieser Lebensphase zum ersten Mal mit psychoaktiven Substanzen (wie Tabak, Alkohol, Marihuana und Ecstasy) in Berührung kommen und dementsprechend teils bewusst, teils unbewusst vielfältige gesundheitsbezogene Einstellungen und Verhaltensweisen erprobt, erlernt, verfestigt oder auch wieder verworfen werden. Die überwiegende Anzahl der im Jugendalter »erfolgreich « angenommenen Verhaltensweisen und Gewohnheiten werden im Erwachsenenalter fortgeführt und sind damit bestimmende Faktoren für den weiteren Gesundheitszustand. Bei ei- ner Betrachtung des Substanzkonsums ist aber nicht nur an die langfristigen Folgen zu denken. So ist die gesundheitliche Situation bereits im Jugendalter $\mathrm{zu}$ einem nicht geringen Ausmaß durch das individuelle Risikoverhalten bestimmt.

Besonders problematisch ist der Substanzkonsum, wenn er sehr früh oder exzessiv einsetzt oder wenn er in Kombination mit anderen problematischen Verhaltensweisen auftritt und daraus eine instrumentelle Gewohnheit wird, die zu einer frühen Einschränkung des Verhaltensrepertoires bei Problemen und Anforderungen führt. So kann regelmäßiger oder exzessiver Konsum von Tabak und Alkohol gerade im Jugendalter $\mathrm{zu}$ schweren Entwicklungsstörungen führen; physiologische Effekte ebenso wie organische Schäden treten schneller als bei Erwachsenen ein, und auch die Zeitspanne vom Missbrauch bis hin zur Abhängigkeit ist gegenüber dem erwachsenen Organismus verkürzt. Im Folgenden werden die wichtigsten Ergebnisse in Bezug auf den Substanzkonsum im europäischen Vergleich vorgestellt. Im Mittelpunkt steht dabei die Frage, wie es um die deutschen Kinder und Jugendlichen bestellt ist.

\section{Tabakkonsum}

Im internationalen Vergleich befinden sich deutsche Jugendliche in der Spitzengruppe der regelmäßigen Tabakkonsumenten. So geben 